\title{
A Cross Sectional Study To Assess The Quality Of Life Of Patients Undergone Surgery For Head And Neck Cancer \\ *Toney Robert \& *** Nila KM
}

\section{Abstract}

Objectives: To determine the Qol of patient who had undergone H\&NCa surgery and to find out the association between Qol after H\&NCa surgery and selected variables. Method: Descriptive surveydesign. Results: low Qol was observed in 45\% subjects. Significant association was found between QoL with many symptoms. Conclusion: With advanced cancer treatment methods, the numbers of H\&NCa survivors are increasing but their Qol would be affected significantly if these problems are not properly addressed. Key words: H\&NCa-Head and Neck Cancer, QoL-Quality of Life, symptom sub-scales.

\section{Introduction}

In daily life, we often do not realize how important simple things like Swallowing, speaking, and eating are for us. Only if a severe disease, such as a head and neck neoplasm, deteriorates these functions do we appreciate the importance of oral health and the related QoL.1H\&NCa describes neoplasms arising from the mouth ( oral cavity), voice box (larynx), throat/upper gullet (pharynx),

salivary glands, nose and sinuses, primary bone tumors of the jaw and middle ear.2

\section{Objectives}

- To determine the quality of life among patients who had undergone head and neck cancer surgery.

- To find out the association between quality of life after head and neck cancer surgery with selected variables.

\section{Materials and method}

Data were collected from forty $\backslash$ patients who had undergone surgery for $\mathrm{H} \& \mathrm{NCa}$ using EORTC- HN35 questionnaire in disease stage and treatment modality(i.e. surgery, chemotherapy and radiotherapy).

\section{Results}

Among the 18 symptoms, weight loss (55\%) was the problem faced by majority of subjects. Other major symptoms were: opening mouth ( $42.5 \%)$, speech problem (35\%), dry mouth (35\%), sticky saliva (30\%) \&coughing (30\%),(0.031) sense loss,(0.033) weight loss.

\section{Figure 1: Distribution of QoL}

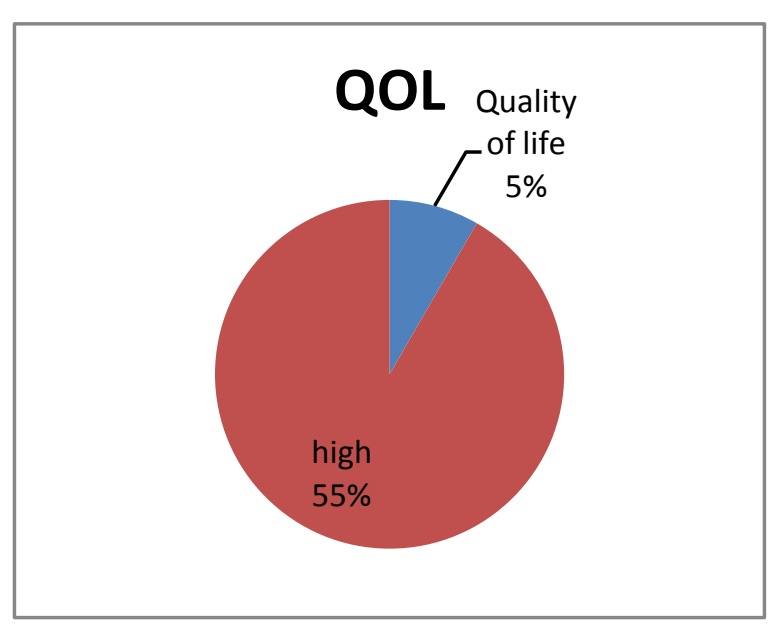


Relationship between QoL and symptoms of subscale showed that there isnegative correlation between them exceptpain and weight gain. Among 18 symptomsub-scales; dry mouth, swallowingproblem, sense problem, speech problem,trouble with social eating, trouble withsocial contact were having very highnegative correlation value $(\mathrm{p}=0.000)$.

\section{Conclusion}

The literature shows that pain management is always been the cornerstone of treatment after surgery but our data suggests that a significant percentage of subjects suffer from other problems at completion of 6 months after treatment.A simple and explicit questionnaire, as used in our study could help in quickly screening for the symptom burden and QoL in this group of patients and this would definitely help in delivery of better symptom directed therapies and achieving therapeutic goal

\section{References}

1. Susanne Singer et al. Assessing and (improving Quality of Life in Patients with Head and Neck Cancer. Available from: http://meetinglib rary.asco.org/content/248-132

2. Key findings for England and Wales for the audit period November 2011 to October 2012 Available from: www.hscic.gov.uk/clinicalaud its

3. Kulkarni MR. Head and Neck Cancer Burden in Ind ia. Int J Head and Neck Surg 2013; 4(1):29-3 5.

4. Aaronson NK et.al. The European Organization for Research and

Treatment of Cancer QLQ-C30: A qualityof-life instrument for use in international clinical trials in oncology. Journal of the National Cancer Institute1993; 85: 365-376

5. Gandhi.AK, RoyS, ThakarA, SharmaA, MohantiB K. Symptom Burden and Quality of Life in Advanced Head andNeck Cancer Patients: AIIMS Study of100 Patients. Indian J Palliate Care.2014 Sep-Dec; 20(3): 189-1 93

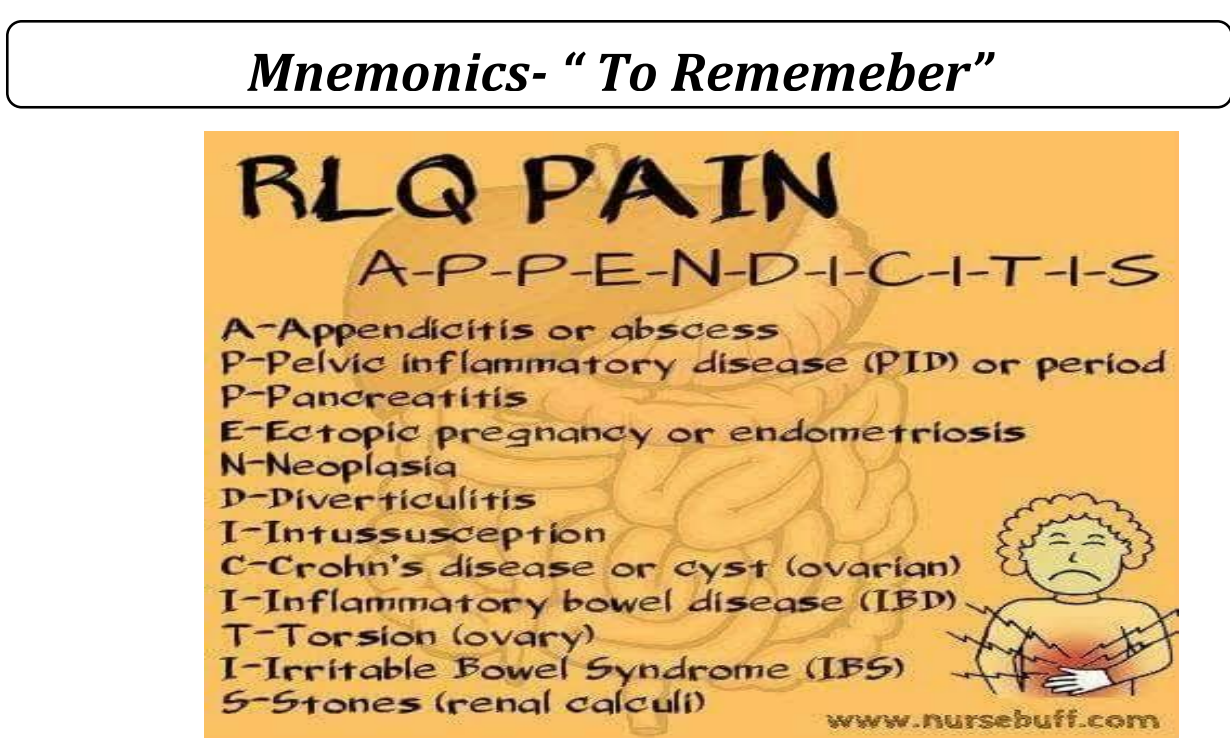

